\title{
Bispectral Index Values of Natural Sleep and its Use in Sleep Endoscopy
}

\author{
Gokhan Yalciner ${ }^{1}$, Mehmet Babademez ${ }^{2}$, Hayati Kale ${ }^{3}$, saliha atalay², Ebubekir Özer², \\ kemal delioğlu ${ }^{4}$, Ayça Dumanlı Özcan ${ }^{1}$, and togay müderris ${ }^{5}$ \\ ${ }^{1}$ Ankara City Hospital \\ ${ }^{2}$ Yildirim Beyazit Universitesi \\ ${ }^{3}$ Medicana International Istanbul Hospital \\ ${ }^{4}$ Basaksehir Cam and Sakura City Hospital \\ ${ }^{5}$ Bakircay Universitesi
}

December 13, 2020

\begin{abstract}
OBJECTIVE: To investigate the bispectral index values of normal sleep stages PATIENTS and METHOD: Simultaneous polysomnography and bispectral index recordings of 21 patients who underwent polysomnography for diagnosis of obstructive sleep apnea were analyzed for determining the BIS values of normal sleep stages. RESULTS: BIS values are found sensitive in reflecting the various stages of natural sleep except for REM and stage N2. REM and stage N2 BIS values were close to each other. CONCLUSION: BIS is sensitive for the evaluation of the stages of natural sleep and BIS values decreases as the sleep stages becomes deeper
\end{abstract}

\section{INTRODUCTION}

Bispectral Index (BIS) monitoring is an objective measurement tool for sedation depth, which is derived from the electroencephalogram (EEG) ${ }^{(1-2)}$ A BIS monitor automatically calculates and displays the BIS index, a dimensionless number, whose value is comprised between 0 (isoelectric EEG waves) and 100 (awake) ${ }^{(3)}$. In non-anesthetized patients, the BIS varies from 90 to 100. On the other hand, total suppression of cortical electrical activity results in a BIS of zero ${ }^{(2)}$.A BIS between 40 and 60 is associated with a low probability of intraoperative awakening and awareness ${ }^{(2)}$.

Most studies evaluating the BIS system have been performed in anesthesia and intensive care units in order to estimate the level of sedation and anesthesia ${ }^{(3)}$. Only a few small studies have investigated the BIS index during natural sleep ${ }^{(4-5-6)}$, and their results are inconsistent.

Drug induced sleep endoscopy (DISE) using sedative agents infusion, guided by a BIS monitor, has been used for decades to directly examine the upper respiratory tract for detecting airway collapse sites ${ }^{(7-8)}$. According to Traxdorf $\mathrm{M}^{*} 7^{*}$ et all for DISE ,BIS leads to a precisely controllable depth of sedation . The DISE-Target controlled infusion of sedative agent-BIS analysis procedure is a step towards a required reproducible protocol of sleep endoscopy capable of standardization. According to Shlemer $\mathrm{JW}^{*}{ }^{*}$ et all . between the commonly used agents for DISE dexmedetomidine's mechanism of action appear most likely to induce natural sleep pathways compared to propofol and midazolam. Different sedation levels such as 50-60 (deep sedation) or 65-75 (light sedation) are recommended for DISE by different researchers ${ }^{(9-10)}$, but it is not exactly known which BIS level corresponds to which phase of normal sleep. 
In this study, we aimed to investigate the BIS values of normal sleep stages, by investigating BIS values during sleep in patients who underwent polysomnography in the sleep laboratory.

\section{PATIENTS AND METHOD}

Twenty-one patients (15 men, 6 women) aged between 36 and 73 years (mean 59.2 years) who underwent polysomnography (PSG) for the diagnosis of obstructive sleep apnea syndrome in the sleep laboratory of Atatürk Training and Research Hospital, between October 2016 and March 2017 were included in this prospective study. Patients with moderate or severe apnea (apnea-hypopnea index [?] 15 ) and the patients who have comorbid systemic illness (such as chronic obstructive pulmonary disease, heart failure)that we think may affect sleep architecture were excluded from study. Local ethical committee approval and written informed consents from patients were obtained.

Simultaneous PSG and BIS recordings of all patients during whole night were acquired.

All PSG recordings were performed with the Philips Respironics Alice 6 Equipment under supervision of experienced sleep technicians and all recordings were manually scored by the same experienced and certified medical doctor (GY). Sleep state scorings were performed according to "AASM Manual for the Scoring of Sleep and Associated Events", version 2.2.

BIS monitoring was performed with the BIS vista monitor (Covidien Medtronic VISTA application revision 3.20 , vista platform revision 2.03). BIS Quatro electrodes were used for recordings.

BIS and PSG recordings were started simultaneously by using the internal clocks of the devices. By the reason of the fact that BIS device records at 1 minute intervals and the epochs in PSG are 30 seconds, 1 BIS value for 2 EEG epochs were obtained and used for analysis.

Only sleep stages lasting 2 minutes or more were included in the analysis (stable sleep / awake state). BIS signals with a signal quality index (SQI) $<50$ were not analyzed.

\section{STATISTICAL ANALYSIS}

The statistical analysis was carried out with SPSS v 23.0 for Windows (SPSS, Chicago, IL) and statistical significance was determined at $\mathrm{p}<0,05$. One-way ANOVA test was used to evaluate BIS averages of SQI groups and comparison of sleep stages according to BIS score averages of SQI values over 70. Post hoc Tukey test was used to compare variables that were found significant.

\section{RESULTS}

Polysomnographic sleep findings of patients were analyzed for the distribution of sleep stages and other sleep related data.Sleep stages were classified as W (wake), N1 (non-rem1), N2 (non-rem2), N3 (non-rem3) and $\mathrm{R}$ (rem). Total recording time, total sleep time, sleep efficiency and the duration of each sleeping stage (W, N1, N2, N3,R) were calculated from the PSG reports (Table 1).

The BIS analysis was performed according to different SQI values. Initially SQI values were divided into three groups (Group 1: 50-70, Group 2: 70-89, Group 3: >90) to calculate BIS averages for each group. When the mean BIS values of these three SQI groups were compared, no significant difference was found between Group 2 and Group 3 as a result of binary comparison of BIS values. There was statistically significant difference between Group 1 and the other groups (Table 2). So we included only the BIS values with an index of SQI over 70 for comparison of BIS values of sleep stages (Table 3).

When the sleep stages were compared, no statistically significant difference was found between the BIS score averages (for SQI values over 70) in stage 2 and REM stages. In all of the other comparisons, there was significant difference in BIS values between the sleep stages (Table 4)

\section{DISCUSSION}

To our best knowledge, this study is one of the few researches that attempt to define BIS values during normal sleep in the sleep laboratory while performing PSG for obstructive sleep apnea syndrome (OSAS) 
diagnosis. Although the patients included in this prospective study were patients suspected to have OSAS; moderate and severe OSAS patients were not included to study to provide a population closer to normal. By this way, a study population with mildly distorted sleep architecture was created.

Only the BIS and sleep stage values in the sleep period that were longer than 2 minutes were taken into account (stable sleep stage) after the end of second minute.

Although the BIS devices are not calibrated to identify the natural sleep stages, there are few studies in the literature investigating the BIS values during normal sleep with conflicting results. In 1999, Slergh et al ${ }^{(6)}$ claimed that; changes in the depth of natural sleep are reflected sensitively by changes in the BIS and BIS may be a simple indicator of the depth of sleep. But their study group was consisted of only 5 subjects. Benini et al ${ }^{(5)}$ also concluded that BIS is sensitive in reflecting the changes on the EEG trace that accompany various stages of natural sleep. To be more specific, they found a progressive decrease in BIS values as the stages of sleep becomes deeper, in their study performed with 15 children. Contrarily, Neuwenhuijs D et al (11) reported that the processed EEG measurements (both BIS and spectral edge frequency) have a limited ability to estimate classical sleep stages as a result of their work with 10 patients.

It has been shown that, BIS can identify stage N3 with satisfactory sensitivity and specificity, but it is unable to distinguish light sleep stage (N1) from REM, sleep or wakefulness (W). ${ }^{(3)}$ Signal quality index is used in different scientific areas to explain some characteristics of determined signals. Therefore the defined SQI is related with signal strength.The SQI is ranged between 0 and 100\% (best signal quality). Mohammad et al suggested that BIS signals should be read in relation to the current signal quality index (SQI) and impedance.

(3) However there is no validated threshold for the quality of the BIS signal and it is not completely clear how the SQI is calculated and what should be an ideal value.

In our study, we first investigated the BIS scores for SQI values of 50-70, 70-90 and over 90 and we found that there was no statistically significant difference between average BIS values of 70-90 and over 90 SQI values, but there was significant difference between 50-70 and 50-70/over 90 SQI values. So we included only the BIS values with an index of SQI over 70 to study.

According to our study, BIS values decrease progressively as the stages of sleep becomes deeper. Mean BIS values were 87.70, 83.94, 74.41, 56.60 and 74.90 For W, N1, N2, N3 and R3 stages respectively (table 4). Furthermore, BIS values were sensitive in reflecting the stages of natural sleep except REM and N2; REM and stage N2 BIS values were similar. One possible explanation of proximity of the index scores in N2 and REM stages may be the inability of the available BIS monitor to distinguish the brain's electrical activiy in these two stages. Our results are incompatible with some of the previous studies, which reported BIS is not $100 \%$ specific in predicting intraoperative awakening and recall ${ }^{(2)}$. In their study, Duarte et al also claimed that the model of BIS monitor could influence the interpretation of the results. We found a statistically significant relationship between BIS scores and sleep stages, which may due to use of a higher model of BIS monitor.

All of the currently available monitors need different period of times to calculate and update the index in response to changes in the depth of anesthesia ${ }^{(2)}$. The time to update BIS records can range from 14 to 155 seconds. For this reason, to acquire more reliable results, we evaluated only the data obtained after the second minute of stable stages.

BIS monitoring may be a practical method to identify sleep stages.Because in oder to recognize sleep stages,BIS is a numerical method that everyone can evaluate in stead of EEG which can only be assesed with a specific training. Which BIS values should be used for sleep endoscopy is not exactly known yet. As a result of their research Babar-Craig *12* et all reported that : average BIS values ranging from 50 to 61 is appropriate for sleep nasendoscopy. Our results suggest that, the 50-70 BIS values correspond to the deep stages of sleep (N3), and it is well known fact that respiratory events are less common in N3 phase of sleep (13). Thus, we recommend that sleep endoscopy should be done at 70-80 BIS values, which corresponds to N1 and N2 phases of sleep where respiratory events are more frequently seen. 
In conclusion, according to us BIS is a usefool tool for distinguishing of normal sleep stages except the R2 REM stages and BIS values decreases as the sleep stages become deeper.

\begin{tabular}{|c|c|c|c|c|c|}
\hline & $\mathrm{N}$ & Mean & Std. Deviation & Minimum & Maximum \\
\hline $\begin{array}{l}<70 \\
70-89 \\
>=90 \\
\text { Total }\end{array}$ & 446150413283278 & $82,8473,8373,2274,81$ & $13,973 \quad 16,702 \quad 16,599 \quad 16,622$ & 27273827 & 98949898 \\
\hline
\end{tabular}

Table 1: Distribution of BIS averages according to SQI

\begin{tabular}{llll}
\hline SQI group (A) & SQI group (B) & Mean Difference (A-B) & p \\
\hline$<70$ & $70-89$ & $9,016^{*}$ & 0,00 \\
& $>=90$ & $9,623^{*}$ & 0,00 \\
$70-89$ & $<70$ & $-9,016^{*}$ & 0,00 \\
& $>=90$ & & \\
& &, 607 & 0,58 \\
$>=90$ & $<70$ & $-9,623^{*}$ & 0,00 \\
& $70-89$ &,- 607 & 0,58 \\
\hline
\end{tabular}

Table 2: BIS averages of SQI groups Table 3: Distribution of BIS score averages of SQI values over 70 according to sleep stages

\begin{tabular}{|c|c|c|c|c|c|}
\hline & $\mathrm{N}$ & Mean & Std. Deviation & Minimum & Maximum \\
\hline $\mathrm{W}$ & 480 & 87,70 & $7,444 \quad 8,614 \quad 13,088 \quad 16,6039,630 \quad 16,615$ & 52 & 98 \\
\hline 1 & 406 & 83,94 & & 47 & 97 \\
\hline 2 & 902 & 74,41 & & 35 & 96 \\
\hline 3 & 722 & 56,60 & & 27 & 95 \\
\hline $\mathrm{R}$ & 3222832 & 74,90 & & 36 & 95 \\
\hline Total & & 73,54 & & 27 & 98 \\
\hline
\end{tabular}

Table 4: Comparison of sleep stages according to BIS score averages of SQI values over 70

\begin{tabular}{llll}
\hline Stage $(\mathbf{A})$ & Stage $(\mathbf{B})$ & Mean Difference $(\mathrm{A}-\mathrm{B})$ & $\mathbf{p}$ \\
\hline $\mathrm{W}$ & 1 & $3,759^{*}$ & 0,00 \\
2 & & \\
3 & & \\
$\mathrm{R}$ & & 0,00 \\
& $13,294^{*}$ & 0,00 \\
& $31,102^{*}$ & 0,00
\end{tabular}




\begin{tabular}{|c|c|c|c|}
\hline Stage (A) & Stage (B) & Mean Difference (A-B) & $\mathbf{p}$ \\
\hline \multirow[t]{7}{*}{1} & $\mathrm{~W}$ & $-3,759^{*}$ & 0,00 \\
\hline & 2 & & \\
\hline & 3 & & \\
\hline & $\mathrm{R}$ & & \\
\hline & & $9,535^{*}$ & 0,00 \\
\hline & & $27,343^{*}$ & 0,00 \\
\hline & & $9,040^{*}$ & 0,00 \\
\hline \multirow[t]{7}{*}{2} & $\mathrm{~W}$ & $-13,294^{*}$ & 0,00 \\
\hline & 1 & & \\
\hline & 3 & & \\
\hline & $\mathrm{R}$ & & \\
\hline & & $-9,535^{*}$ & 0,00 \\
\hline & & $17,807^{*}$ & 0,00 \\
\hline & &,- 495 & 0,97 \\
\hline \multirow[t]{7}{*}{3} & W & $-31,102^{*}$ & 0,00 \\
\hline & 1 & & \\
\hline & 2 & & \\
\hline & $\mathrm{R}$ & & \\
\hline & & $-27,343^{*}$ & 0,00 \\
\hline & & $-17,807^{*}$ & 0,00 \\
\hline & & $-18,302^{*}$ & 0,00 \\
\hline \multirow[t]{7}{*}{$\mathrm{R}$} & $\mathrm{W}$ & $3,759^{*}$ & 0,00 \\
\hline & 1 & & \\
\hline & 2 & & \\
\hline & 3 & & \\
\hline & & $13,294^{*}$ & 0,00 \\
\hline & & $31,102^{*}$ & 0,97 \\
\hline & & $12,799^{*}$ & 0,00 \\
\hline
\end{tabular}

\section{Abbreviations}

Associate Professor Gökhan Yalçıner M.D: GY

Bispectral Index: BIS

Drug induced sleep endoscopy: DISE

Electroencephalogram: EEG

Obstructive sleep apnea syndrome: OSAS

Polysomnography: PSG

Signal quality index: SQI

\section{REFERENCES}

1. Park Sw, Lee H, Ahn H. Bispectral Index Versus Standart Monitoring In Sedation For Endoscopic Procedures: A Systematic Review And Meta-Analysis. Dig Dis Sci. 2016 Mar;61(3):814-24

2. Duarte LT, Saraiva RA. When The Bispectral Index (BIS) Can Give False Results. Rev Bras Anestesiol. 2009;59(1):99-109. 
3. Benissa MR, Khirani S, Hartley S, Adala A, Ramirez A, Fernandez-Bolanos M, Quera-Salva MA, Fauroux B. Utility Of The Bispectral Index For Assessing Natural Physiological Sleep Stages In Children And Young Adults. J Clin Moint Comput 2016 30:957-963.

4. Dahaba AA, Xue JX, Xu GX, Liu QH, Metzler H. Bilateral Bispectral Index (BIS)- Vista As A Measure Of Physiologic Sleep In Sleep-Deprived Anesthesiologists. Minerva Anestesiol 2011;77:388-93.

5. Benini F, Trapanotto M, Sartori S, Capretta A, Gobber D, Boniver C, Zacchello F. Analysis Of The Bispectral Index During Natural Sleep In Children. Anesth Analg, 2005;101(3):641-4.

6. Sleigh JW, Andrzejowski J, Steyn-Ross A, Steyn-Ross M. The Bispectral Index: A Measure Of Depth Of Sleep? Anesth Analg, 1999;88(3):659-61.

7. Traxdorf M, Tschaikowsky K, Scherf C, Bauer J, Iro H, Angerer F. Drug-Induced Sleep Endoscopy (DISE) with Target Controlled Infusion (TCI) and Bispectral Analysis in Obstructive Sleep Apnea. Eur Arch Otorhinolaryngol. 2012 Apr;269(4):1277-9. doi: 10.1007/s00405-011- 1798-1.

8. Shteamer JW, Dedhia RC. Sedative Choice in Drug-induced Sleep Endoscopy: A NeuropharmacologyBased Review. Laryngoscope 2017 Jan;127(1):273-279. Doi:10.1002/lary.26132. epub 2016 Jul 1.

9. Stierer TL, Ishman SL. Bispectral Index In Evaluating Effects Of Sedation Depth On Drug-Induced Sleep Endoscopy: Dise Or No Dice. J Clin Sleep Med 2015 Sep 15;11(9):965-6.

10. Lo YL, Ni YL, Wang TY, Lin TY, Li HY, White DP, Lin JR, Kuo HP. Bispectral Index In Evaluating Effects Of Sedation Depth On Drug-Induced Sleep Endoscopy. J Clin Sleep Med. 2015 Sep $15 ; 11(9): 1011-20$

11. Nieuwenhuijs D, Coleman EL, Douglas NJ, Drummond GB, Dahan A. Bispectral Index Values And Spectral Edge Frequency At Different Stages Of Physiologic Sleep. Anesth Analg. 2002;94:125-9 